\title{
Focus and the LF of NP quantification"
}

\author{
Elena Herburger \\ University of Southern California
}

\section{The Definiteness Effect (Milsark 1977):}

Milsark (1977) observes that some NPs are acceptable in thereinsertion contexts while others are not, as shown in (1). He distinguishes them by the terms weak and strong.

(1) a. There are some/three/many/few/no children in the garden

b. There is (are) every/each/all/most child(ren) in the garden

He also notes that the determiners introducing weak NPs have a special, what he calls 'cardinal' reading. For instance, $f e w$ in few children in (1a) does not mean the same as $f e w$ in the partitive NP few of the children; whereas in the latter the determiner picks out a certain proportion of the children, in the former it means something like 'few in number'.

Milsark shows, however, that once we change the environment, it becomes possible to interpret $f e w$ in $f e w$ children very much like $f e w$ in a partitive NP. Such a reading is optionally available if the NP is the subject of a so-called Stage Level (SL) predicate, as in (2a):

(2) a. Few children are playing

Here few children is ambiguous. It can be interpreted as 'children who are few in number' or in a manner very similar to the overt partitive 'few of the children'. On the other hand, when the predicate is interpreted as a property-denoting Individual Level (IL) predicate, then the cardinal reading is absent and only the partitive-like reading is available. ${ }^{1}$ For instance, in:

\footnotetext{
- I am grateful to Barry Schein for generous support and immensely helpful advice. Thanks also to Juan Uriagereka, Anna Szabolcsi, Jean-Roger Vergnaud, Jose Camacho, Liliana Sanchez, Tim Stowell and Richard Larson for their comments and encouragement.

${ }^{1}$ Milsark actually uses the terms state descriptive predicate and property predicate. The former refers to 'conditions in which an entity finds itself and which are subject to change without there being any essential alteration of the entity' (p. 12). The latter describes 'some trait possessed by the entity and which is assumed to be more or less permanent, or at least to be such that some significant change in the 
b. Few children like spinach

few children can only be understood along the lines of 'few of the children' but not as 'children who are few in number'. Unlike NPs introduced by the determiners $f e w$, some, etc., NPs introduced by the determiners most, all, every, each, neither only have strong, partitive-like readings.

The reason I am using partitivity to describe the strong readings, rather than the terms proportional or non-cardinal (cf. Partee (1988) e.g.), is because of determiners like five. This determiner is never proportional and always cardinal, but it nonetheless participates in the same ambiguity as few, cf. (3).

(3) a. Last year, five babies were born

In (3a) the predicate is SL and the NP reads as 'babies five in number'. On the other hand, in

b. Last year, five babies were newborns

the predicate is an IL predicate and we only obtain a reading of the subject equivalent to 'five of the babies'. An interpretation of the subject as 'babies five in number' is not available.

In order to capture the intuition that the determiners of weak NPs receive a special cardinal reading, Milsark proposes that these determiners are in fact non-quantificational cardinality predicates. He then suggests that weak NPs in there-insertion sentences receive their quantificational force from an existential quantifier hidden in the expletive there. Since strong NPs are considered to be truly quantificational, they are ruled out in there-insertion contexts. It is left as an open question how weak NPs obtain their quantificational force when they do not appear in there-insertion sentences but rather in SPEC of IP, as in (2a) for example. Regarding the generalization that weak NPs cannot be the subject of IL predicates, Milsark suggests that only quantificational (strong) NPs make good 'topics', and that being a topic is a prerequisite for allowing for IL predication.

The question of how weak NPs generally receive their quantificational force is one of the central issues discussed in Diesing

character of the entity will result if the description in altered.' (p.13) I will return to the distinction between two types of predication later and for the time being merely use SL and IL as descriptive labels. 
(1990). She adopts Milsark's analysis of the strong/weak contrast and assumes that unlike strong NPs weak NPs are non-quantificational and receive their quantificational force through existential closure. Extending the scope of Milsark's analysis so as to also account for those weak NPs which do not occur in there-insertion sentences she argues, first, that all weak NPs appear inside the VP at LF and, second, that it is the VP which is a domain of existential closure.

Evidence for the claim that all weak NPs appear inside VP at LF is derived from scrambling in German. Diesing argues that NPs that have scrambled out of the VP receive a strong interpretation, while NPs that remain inside VP receive a weak interpretation. Based on the assumption that German wears its LF on its sleeve, she takes the correlation between an NP being weak and its occuring inside VP at S-structure in German to be indicative of which position weak NPs in English take at LF. As a consequence of this she proposes those weak NPs that appear in Spec of IP at S-structure in English lower back into the VP at LF.

The claim that the VP is a domain of existential closure is made in connection with the hypothesis that quantified NPs are interpreted in 'tripartite structures'. In particular, Diesing proposes that at a level after LF, quantified NPs are mapped into a tripartite structure consisting of 'operator', 'restrictive clause' and 'nuclear scope', where material outside the VP is mapped into the restrictor and material inside the VP is mapped into the nuclear scope (=Mapping hypothesis). Moreover, she assumes that the nuclear scope is the place where existential closure takes place. Since weak NPs are viewed as non-quantificational, following the lines of Milsark's analysis, Diesing argues that weak NPs, unlike strong NPs, are not split apart into a tripartite structure but that they are instead left intact and interpreted inside the scope of existential closure.

The Milsark-type of analysis of weak NPs runs into two problems. The second one will be laid out in some detail in the next sections. The first one can be stated briefly: existential closure cannot be the general source of the quantificational force of weak NPs. If it were then it would be predicted that each and every weak NP be monotone increasing. ${ }^{2}$ The reason is that if weak NPs obtain their quantificational force from existential closure, as illustrated in

(4) E x: some/three/many/few/no (x) \& children (x) in the garden (x)

\footnotetext{
${ }^{2}$ A determiner is monotone increasing (in its first argament) if it holds that if [D $A$ ] $B$ then [D $\left.A^{\prime}\right] B$ when $A C A^{\prime}$. It is decreasing if the opposite is true: if [D A] $B$ then [D A'] $B$ when $A^{\prime} C A$.
} 
then they should behave like other existentially quantified NPs, which means that they should be monotone increasing. However, this is clearly false because of weak NPs introduced by decreasing determiners like few, no, and at most five (see Schein 1992, BenShalom 1993, Beghelli 1993 on maximality). Thus, the view that all weak determiners are cardinal predicates and that weak NPs are bound by existential closure wrongly predicts that $f e w$, for instance, which is decreasing, is semantically undistinguishable from $a f e w$, which is increasing. Therefore we have to abandon the idea that there is a process of existential closure for weak NPs and go back to the view that even the determiners of weak NPs are quantificational, just like those of strong NPs. This means that the ambiguity of the determiners $f e w$ and many is an ambiguity between two genuinely quantificational readings, one proportional and one symmetric. ${ }^{34}$

The second problem is the following: the ambiguity of determiners like $f e w$ and many gives rise to a distinction between NPs that actually does not coincide with the strong/weak distinction. For if we take into account the effects of focus inside quantified NPs, we will see that there are weak NPs that are introduced by determiners that are not symmetric but proportional. This suggests that the ambiguity of determiners like $f e w$ and many is independent of the Definiteness Effect and that it is necessary to look somewhere else for a criterion that allows us to tell apart weak from strong NPs.

In what follows, I assume that it can indeed be argued that weak NPs appear inside the scope of the VP at LF while strongs do not. Because of the way focus inside quantified NPs affects their semantics, however, I will look for an alternative to the Milsark-type

\footnotetext{
${ }^{3}$ According to Barwise and Cooper (1981), a determiner is symmetric iff [Q A] B <-> [Q B] A. Moreover, (given that it is conservative) a determiner is symmetric iff it is intersective, i.e. [Q A] B $\leftrightarrow[Q, \cap B]$ B. Determiners like $f e w$ and many are ambiguous between a symmetric and a proportional reading. When they are symmetric, it only matters how many things are both $A$ and $B$. For instance, symmetric few requires that the number of things that are both $A$ and $B$ is relatively small, but it is irrelevant whether actually all things that are $A$ are picked out so lang as they are also $B$ and $f e w$ in number. On the other hand, this is not true of proportional $f e w$. Unlike symmetric $f e w$, it requires that only a contextually determined small proportion of things that are $A$ are also $B$. Here the number of things that are $A$ is also relevant not just the number of things that are in the intersection of $A$ and $B$.

${ }^{4}$ One could try to argue that decreasing determiners do not really pose a problem for existential closure, if they can be decomposed into NEG and an increasing determiner. For instance, no would then convert into NEG and some, $f e w$ into NEG and many, at most five into NEG and more than five. However, an approach like this would violate the lexical integrity of determiners.
} 
way of analyzing the weak/strong contrast and, in particular, I argue in the spirit of Enc (1991) that the (covert) partitivity and 'familiarity' of the NP is the crucial divide between weak and strong NPs. In connection with the analysis, empirical arguments are given which show that a traditional view of the logical form of quantified NPs is to be preferred over a tripartite approach. It is also argued that a syntactic implementation of the Prague view of focus is what is needed to account for the data adequately. Finally, a proposal is made concerning scopal properties of subjects which previously have been attributed to a semantic distinction between SL and IL predicates.

\section{Focus-affected readings of weak NPs:}

Focus is known to affect the truth conditions of a sentence when it occurs inside the scope of intentional operators, expressions like only, or adverbs of quantification (Dretske 1972, Rooth 1985, Krifka 1991). Consider (5a) vs. (5b), due to Rooth: (CAPS represent focus.)

(5) a. In St. Petersburg, OFFICERS always escorted ballerinas

b. In St. Petersburg, officers always escorted BALLERINAS

These sentences do not have the same truth conditions. Whereas (5a) asserts that it was always the case that those who escorted ballerinas were officers, (5b) makes a different claim, namely that it was always the case that the escorts of officers were ballerinas. It seems then that the matrix of the adverb of quantification is determined by what is focused. (5a) and (5b) can then be argued to have logical forms like in (6a) and (7a) (cf. Rooth 1985, Krifka 1992): ${ }^{5}$

(6) a. [Always e: $x$ escorted ballerinas at e] OFFICERS escorted ballerinas at $e$

b. [Always e: officers escorted $x$ at e] officers escorted

BALLERINAS at $\mathrm{e}$

Consider next the sentence in (7):

(7) Few INCOMPETENT cooks applied

According to much of the literature on the Definiteness Effect (e.g.

\footnotetext{
${ }^{5}$ In the schema [Q A] B, A represents the restriction or first argument of the generalized quantifier, and $B$ the matrix or second argument.
} 
Milsark 1977, Partee 1988, Diesing 1990), this sentence should have just two readings: one reading where the determiner is cardinality predicate and where the sentence means that the incompetent cooks applying were few in number (= weak reading), and one reading where the determiner is proportional and where the sentence means that few of the incompetent cooks applied (= strong reading) ${ }^{6}$ These readings are given in ( $8 a$ ) and ( $8 b)$, respectively. (In (8a), the NP is bound by existential closure since $f e w$ is treated as a nonquantificational cardinality predicate. The point, however, holds equally if it is treated as a symmetric determiner.)

(8) a. [E $x$ : few (x) \& INCOMPETENT (x) \& cooks ( $x$ )] applied (x)

b. [Few $x$ : INCOMPETENT $(x) \&$ cooks $(x)]$ applied $(x)$

It seems that when (7) is interpreted both as in $(8 a)$ or $(8 b)$ focus is contrastive, contrasting incompetent with competent, and it can also be emphatic. But it has no effect on the meaning or truth conditions of the sentence.

Note now that the re exists a third reading of (7). And in this reading focus actually changes the meaning and the truth conditions of the sentence. In a sense to be made precise, focus here is semantic, not just contrastive or emphatic. This suggests that we need to distinguish between different types of focus, a question we will come back to. The third reading of (7) can be paraphrased as 'few cooks that applied were incompetent.' Its LF is given in (8c):

\section{c. [Few $x$ : cooks $(x) \&$ applied (x)] INCOMPETENT (x)}

Here the determiner of the NP does not have a symmetric reading nor does the NP receive the strong reading in $(8 b)$, where the proportional $f e w$ is restricted by incompetent cooks. However, $(8 \mathrm{c})$ is similar to (8b) in that the determiner is proportional. A crucial difference is that the restriction of the determiner here corresponds to cooks that applied rather than to incompetent cooks and the matrix corresponds to to the focused predicate INCOMPETENT, rather than to the VP. I refer to this type of reading as focus-affected ( $\mathrm{f}-\mathrm{a}$ reading).

It is possible to test whether this reading is really available for (7) by checking whether the sentence can be judged true in a scenario like the one outlined in (9). The prediction is that it can.

\footnotetext{
${ }^{6}$ Barwise and Cooper (1981) actually do not draw this distinction, but see Partee (1988) on how this distinction is implicit in their discussion of the data.
} 
(9) Despite the efforts of its tourist board, there remain as many as 20 incompetent cooks in some small town. They all applied for the same job at the hotel. Besides them, also 80 competent cooks applied for this job.

In (9) actually ALL incompetent cooks applied. At the same time, they made up only a rather small fraction of the total number of applying cooks, namely $1 / 5$. And what's more, they were not few, for they were 20 , which is a considerable number.

If (7) can be true in (9), then it must have as one of its reading the reading represented in $(8 \mathrm{c})$. In other words, it must have one reading where it means that few cooks that applied were incompetent. First, the fact that actually all incompetent cooks applied rules out any analysis of the NP as strong in this reading of (7), cf. (8b). Moreover, the fact that the incompetent cooks applying were numerous rules out a symmetric analysis of the NP where it is introduced by a symmetric $f e w, \mathrm{cf}$. (8a). What remains is an analysis of the NP introduced by $f e w$ as $\mathrm{f}-\mathrm{a}$, as in (8c). It is interesing to note that in (8c) the determiner is proportional and not symmetric. We will return to this.

The characteristic of a f-a reading of an NP is that focus here is semantic and not just contrastive or emphatic. It is semantic in that it causes the focused predicate to function in the same way the VP normally functions, namely as the main predicate of the sentence and the matrix of the determiner. This is stated in (10)

(10) Focus-affected Readings:

Semantic focus inside an NP gives rise to a $f$-a interpretation of that NP, where the focused predicate serves as the main predicate of the sentence and the matrix of the determiner.

Next, we observe that $f$-a readings are restricted to certain contexts. As the data to be discussed show, $f$-a readings appear only in weak NPs, that is, in those NPs introduced by the determiners $f e w$, many, etc. which occur inside VP at LF. F-a readings are clearly impossible in strong NPs, where focus can only be contrastive or emphatic but not semantic in the present sense. This distributional restriction of $f-a$ readings is stated in (11):

(11) Definiteness Effect of $f$-a readings:

Contrastive and emphatic focus can appear both in weak and 
strong NPs. Semantic focus is found only inside NPs that are weak. $^{78}$

The discussion of (7) has shown that when an NP is interpreted in $\mathrm{f}$-a terms the determiner need not be symmetric. The generalization in (11) then predicts that in the core cases of weak NPs, namely there-insertion NPs, a determiner like few or many need not be symmetric but can be proportional instead if the NP contains a focused predicate and receives a f-a interpretation. To see whether this is true compare (12a) with (12b):

(12) a. There are many native speakers of Basque THAT ARE CITIZENS OF SPAIN

b. There are many citizens of Spain THAT ARE NATIVE SPEAKERS OF BASQUE

It seems clear that these sentences do not necessarily have the same truth conditions, for it is possible to judge the first one true and the second one false. But if so, the determiner many here is clearly not symmetric. Rather, it appears that (12a) and (12b) have the $f-a$ readings given in (12c) and (12d), respectively, and that is the reason why their truth-conditions diverge.

c. [Many $x$ : native speakers of Basque $(x) \&$ are $(x)$ ] CITIZENS OF SPAIN (x)

d. [Many $x$ : citizens of Spain (x) \& are (x)] NATIVE SPEAKERS OF BASQUE (x)

(12a) means 'many native speakers of Basque (that exist) are citizens of Spain', which is true. (12b), on the other hand, reads as 'many

\footnotetext{
${ }^{7}$ This generalization recalls Gueron's (1980) discussion of the contrast between definite and indefinite NPs. She argues that at LF indefinite (presentational) subjects appear inside the scope of the verb at LF while definite subjects do not. (In the former case the verb raises, but not in the latter.) Moreover, she claims that focus on indefinite NPs can be contrastive or presentational, while focus on definite NPs can only be contrastive. Although the semantics of presentational focus are not very explicit, it seems that it could be regarded as a case of semantic focus.

${ }^{8}$ If weak NPs and strong NPs are inside and outside the VP at LF respectively, as is assumed here, then (11) can be stated more generally and we can say that focus is semantic only when it occurs inside the VP at LF. Although it does not deal with focus inside quantified NPs, it is interesting to note that Partee (1991) reaches an empirically very similar conclusion. For a discussion of the type of analysis employed in Partee (1991), see section 5 .
} 
citizens of Spain (that exist) are native speakers of Basque'. And that's false. ${ }^{9}$

Note also that parallel to other weak NPs (Milsark 1977, Diesing 1990), f-a weak NPs are interpreted inside the scope of the VP at LF. A relevant example is given in (13):

\section{Every teacher flunked many RICH kids}

This sentence has many readings, but I want to concentrate on its $f$-a interpretation. Consonant with the generalization in (11), such a reading is only possible when many has VP scope. Thus, the sentence can mean that 'for every teacher it is true that many kids that he or she flunked were rich.' However, it does not seem to have a reading where it is interpreted as 'many kids that are such that every teacher flunked them were rich.'

The examples in (12) and (13) show that $f$-a readings can arise in weak NPs. Let us see now whether they can arise ONLY in weak NPs, as is claimed in (11). Compare the following two sentences:

(14) a. Few/many/no/three/some INCOMPETENT cooks applied b. Most/all/every/each INCOMPETENT cook(s) applied

Consonant with the generalization in (11), the NPs in the first sentence, which are weak, can be interpreted in $\mathrm{f}$-a manner, while the NPs in the second sentence, which are strong, cannot. Thus, (14a) can be read for instance as 'few cooks that applied were incompetent'. But (14b) cannot mean 'most cooks that applied were incompetent'. The sole contribution of focus to the meaning of the sentence here is that of contrast or emphasis on incompetent.

Consider also the example in (15):

\section{Few/many/no/three/some COOKS like spinach}

In (15), the NP in question is the subject of an IL predicate, which, as we have seen in section 1. , is necessarily strong in that the NP

\footnotetext{
9 One could perhaps argue that in (12) the focused predicate, i.e. the relative clause, is not inside the NP at S-structure but a sister of it and then suggest that it is because of this syntax that the focused predicate is interpreted as the main predicate. Notice, however, that $\mathrm{f}-\mathrm{a}$ reading arise equally in (i) and (ii), where it is impossible to argue that the focused predicate is not inside the NP at S-structure:

(i) There are many SPANISH Basque speakers

(ii) There are many BASQUE-SPEAKING Spaniards
} 
receives a partitive reading. And again, in accordance with the generalization in (11), focus here can only be contrastive or emphatic, but it cannot be semantic and no $\mathrm{f}$-a reading is available. In particular, (14) cannot mean 'few that like spinach are cooks'. Rather it means something like 'few cooks and not waiters like spinach.'

Further evidence that shows that $\mathrm{f}$-a readings do not arise in strong NPs but only in weak NPs comes from scrambling in German.

(16) a. weil viele SCHWALBEN wohl in den Süden fliegen b. weil wohl viele SCHWALBEN in den Süden fliegen 'because many SWALLOWS probably fly to the South'

Scrambled NPs in German are known to receive strong readings, whereas non-scrambled are argued to pattern with weak NPs. Given (11), we now expect that focus in a scrambled NP can only be contrastive or emphatic, whereas the focus in the non-scrambled NP can be semantic. This prediction is borne out. While (16a), where the NP is scrambled, only allows for the strong reading in $(16 \mathrm{c})$,

c. [Many $x$ : SWALLOWS $(x)$ ] fly to the South $(x)$

d. [Many $x$ : fly to the South $(x)$ ] SWALLOWS $(x)^{10} 11$

\footnotetext{
${ }^{10} \mathrm{Cf}$. also Moltmann (1990), who notes that focns on scrambled NPs is contrastive while focus on non-scrambled NPs is presentational in Gueron's sense. (I should note that Moltmann does not share the intuition that scrambled NPs receive strong or 'specific' readings. In this respect my intuitions do not coincide with hers, but rather with those reported in Kratzer 1989 and Diesing 1990.)

${ }^{11}{ }^{11}$ It is interesting to note non-scrambled NPs can sometimes receive strong readings, eg. when they are focused. Consider (i), adapted from Kratzer (1989):

(i) weil ja viele WILDSCHWEINE intelligent sind

because prtcl. many BOARS intelligent are

because many BOARS are intelligent

It seems to me that focus in (i) can only be contrastive. If we assume the generalization in (11) then the NP in (i) must be outside the VP at LF, which means that it scrambles or QRs at LF. This is consonant with the fact that the NP receives a strong reading and that it is the subject of an IL predicate. Note that if true this suggests that Diesing's claim that German wears its LF on its sleeve is not accurate. Rather, it appears that although German S-structure is 'disambiguating' wrt. to scrambled NPs, which must be strong, it is not disambiguating wrt. to non-scrambled NPs, which can be either weak or strong. The point that is relevant for (16) is clearly that non-scrambled NPs CAN be weak, not that they MUST. Note that if German Sstructure is less revealing than assumed this does not invalidate the claim that weak NPs are inside the scope of VP at LF.
} 
(16b), where the NP surfaces in the VP, also allows for the f-a reading in (16d), meaning 'many things flying to the South are swallows.'

What these data illustrate is that NPs with $f$-a readings have the same distribution as symmetric weak NPs. In fact, they represent a certain kind of weak NP. They clearly differ from other weak NPs in that they receive a reading where the determiners $f e w$ and many are proportional. The data thus show that even in those cases where the distinction between symmetric and proportional determiner is applicable, it does not coincide with the distinction between weak and strong NPs. Rather, in distribution at least, the strong/weak contrasts seems to correlate with whether an NP appears inside VP at LF or not. How this fits in with the claim that the weak/strong distinction has to do with partitivity will be discussed in section 6 . First, however, I would like to turn to the analysis of $\mathrm{f}-\mathrm{a}$ readings. ${ }^{12}$

\section{An L.F analysis of $f-a$ readings:}

The analysis assumes a Davidsonian semantics, specifically the theory of events discussed in Higginbotham (1987). According to this view, all predicates, both so-called SL and IL predicates, have an event argument. This is also true of nouns, even non-eventive ones like $\operatorname{dog}$ (and presumably other predicates). Crucially, I take the Davidsonsian event operator to mark the scope of the VP and for concreteness I assume that it appears in INFL. It follows from the generalization that weak but not strong NPs occur inside VP at LF that the event variable of weak NPs but not that of strong NPs is bound by the event operator. (The latter is bound by the discourse.)

The strictly Davidsonian semantics adopted here is not compatible with Diesing's or similar views of the SL/IL contrast, where event argument places are only accorded to SL predicates but not to IL predicates (nor to nouns) (cf. also Kratzer 1989). We then have to look for an alternative to explain the facts. In descriptive terms, what needs to be accounted for is really the observation that

\footnotetext{
${ }^{12}$ The data presented involve subjects, but the generalizations in (10) and (11) also seem to apply to objects, consider, eg.

(i) I know few FAMOUS skiers

(ii) I know most FAMOUS skiers

As predicted by (10) and (11), (i) can have a f-a reading and (ii) cannot: (i) can mean 'for me it holds true that few of the skiers that $I$ know are famous', but (ii) cannot mean 'for me it holds true that most of the skiers that I know are famous.' Notice that it is irrelevant here that the predicate is $\mathrm{IL}$, for whether a predicate is SL or IL only seems to affect the interpretation of subjects, as shown in Diesing (1990).
} 
IL subjects are always strong, while SL subjects can be strong or weak, where strong and weak correlate with being outside or inside the scope of the VP at LF, or, in our terms, the scope of the event operator. I want to suggest that this does not follow from a semantic difference between predicates where IL predicates have a different argument structure than SL predicates. Rather, the scopal difference between IL and SL subjects derives from a notion of grounding. This proposal owes much to Milsark's (1977) idea that strong but not weak NPs make good 'topics' for sentences where the predicate is IL.

Let us first adopt the functionalist view that a sentence has to be 'about a topic' and, more generally, that the information we aquire about the world is organized in terms of what the information is about. We will then say that a sentence is 'grounded' in an object or an event that meets the description provided by the sentence's topic and, moreover, that the topic takes widest scope in the sentence. There are of course discourse conditions on topics, but we expect that there are also epistemological conditions on the kinds of objects or events that provide the grounding for sentences. The sorts of objects that we think sentences are 'about' will depend on how we are related to these objects. Let us say that

(17) Grounding:

In order for a sentence to be grounded in an event or an NP $\alpha$, where $\alpha$ is the topic of the sentence, it has to as to be in principle possible to have epistemological access to $\alpha .^{13}$

Grounding accounts for the scopal differences of IL vs. SL subjects as follows: so-called IL predicates typically describe events that are as such not epistemically salient to us. Note in this context that verbs of perception do not allow for IL small clauses, see eg. Raposo and Uriagereka (1990). Therefore, IL events are not available for grounding, and we have to ground an IL assertion in something else, namely the subject. In contrast, what are considered SL events are epistemologically salient enough to allow for a sentence to be grounded in them, which ties in with the observation that verbs of perception 'select' SL small clauses. Because of the epistemological salience of SL events, SL assertions can be grounded in the event itself. Nothing, however, precludes that they are grounded in the subject, that is an available option. Thus, the difference between IL

\footnotetext{
${ }^{13}$ Grounding is a notion that is similar to but weaker than Russell's knowledge by acquaintance. It is weaker because it only says that it must be IN PRINCIPLE POSSIBLE to have epistemological access to the thing we are talking about.
} 


$$
\text { [IP E e: apply (e) [VP } \left.\text { [NP }_{\mathrm{NP}} \text { few } x: \operatorname{cooks}(\mathrm{x}, \mathrm{e})\right] \quad 0(\mathrm{x}, \mathrm{e})
$$
INCOMPETENT $(x)$ ]

It reads 'there was an event of applying such that few cooks in it were agents in it and were incompetent.' This is equivalent to saying 'few cooks that applied were incompetent', which is the logical form given in (8c). The 'in it' part results from the existential event operator binding into cooks. The relation $\mathrm{O}$ is the thematic relation that holds between an event and its participants, in this case it is the thematic role of being an agent. ${ }^{15}$

In the context of (19), it is important to note that we must ensure that no subevents are picked when we interpret this type of LF. (This was pointed out to me by Hans Kamp, p.c.). The problem is that when interpreting (19) we can always pick a subevent that is small enough that only few of the many cooks are involved in the subevent and (19) would be vacuously true.

One way we might be able to ensure that a sufficiently large event is picked out in (19) is to substitute the existential operator in (19) with a definite description. This would actually also allow us to maintain that the decreasing determiners of weak NPs are nonquantificational cardinality predicates and that the quantificational force of these NPs derives from existential closure, for by virtue of forcing the event be maximal, we would correctly prevent these NPs from being increasing (Bill Ladusaw, p.c.).

A reason, however, not to follow through with this approach is that in the same way we prevent weak NPs introduced by decreasing determiners from being increasing, we also prevent weak NPs that are introduced by increasing determiners from being increasing, so that we would again be in a position were it is not possible to distinguish between the decreasing $f e w$ and the increasing $a f e w$, only that now we would say that neither is increasing.

A second reason not use a definite description so as to preclude the picking of subevents is that the problem is fully general. It also arises in cases where the conditions for definite reference are lacking, see Schein (1992). Thus, (20) is vacuously true if the adverb quantifies over events or times.

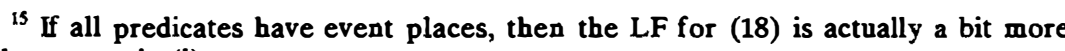
elaborate, as in (i):

(i) [E e: applied (e) [[ few $x$ : cooks $(x, e)])(x, e) E$ e': $R\left(e, e^{\prime}\right)$ \& INCOMPETENT $\left.\left(\mathbf{x}, \mathbf{e}^{\prime}\right)\right]$
} 
and SL assertions reduces to whether it is an event description or the subject which is put forth as the topic of the sentence.

The analysis as such consists in the following claim: a focused predicate inside a VP-internal NP extraposes at LF. Thereby it moves to a position where it mutually c-commands the VP internal subject position and it becomes the main predicate. ${ }^{14}$ This is shown in (18), the $L F$ for the $f-a$ reading of (7):

(7) Few INCOMPETENT cooks applied

(8) c. [Few x: cooks (x) \& applied (x)] INCOMPETENT (x)

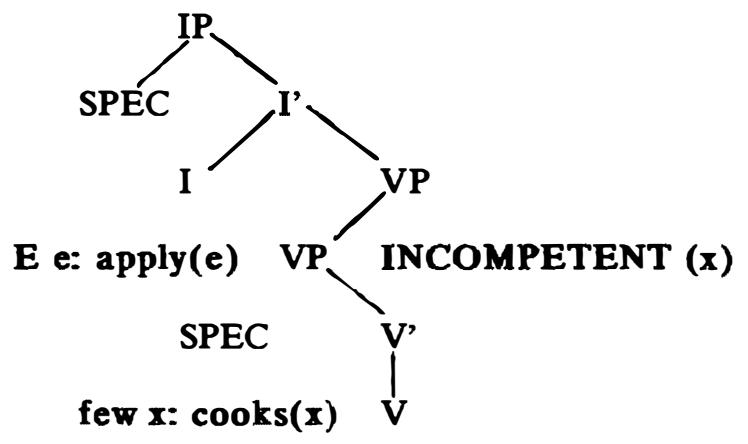

The question that immediately arises is how does this analysis derive the effect that the verb appears in the restriction of the determiner in (8c). In other words, how do we obtain a logical form 'few cooks that applied' rather than 'few cooks' without doing violence to the syntax and actually moving the verb into the $N$ ' at LF or removing the determiner from the NP?

The answer comes rather directly from the Davidsonian semantics adopted here. According to this view, the event operator binds the event variable of the verb in the LF of (7), asserting the existence of an event of applying. It also binds the event variable of the NP introduced by $f e w$, which means that the NP refers to things that are $\mathbf{N}$ in the event. But if so, we have actually arrived at the equivalent of applied literally joining the restriction. Consider (19):

\footnotetext{
${ }^{14}$ I a $\mathrm{m}$ adopting the view here that nodes created by adjunction do not create barriers for government, following May (1985). It is also assumed that Left Branch Extractions are licit at LF.
} 
Once, noone knows when, noone arrived

Since (20) is sensibly used, we must allow the context to restrict the event in such a way that we pick a sufficiently large one. One cannot simply take the adverb to be a definite description, because, as the parenthetical makes clear, the condition for definite reference are not met in (20). But if we rely on the context to pick a sufficiently large event in (20), there is no reason to suppose that a similar contextual restriction does not also operate in (19). Therefore, let us elaborate (19) as in (21), where $C$ denotes a relevant context:

(21) [E e: C (e) \& apply (e)] [few x: cooks $(x, e)]$ E e': R (e, e') \& INCOMPETENT $\left(x, e^{\prime}\right)$

\section{A corollary: a syntactic defintion of restriction and matrix}

One consequence of the account of $f$-a readings of NPs presented is that it suggests that it is not the catergorial labels N' and VP which determine restriction and matrix of a determiner. Rather, it is the syntactic configuration of the sentence. Restriction and matrix are now defined as in (22):

(22) Matrix and restriction of a generalized quantifier:

The matrix is the structural main predicate of the sentence, the restriction is what is c-commanded by the determiner.

Most of the time, (22) will give us N' as the restriction, and the VP as the matrix, but not always. It crucially will not when focus changes the syntax of the sentence.

With this in mind, let us turn to some data discussed in Westerstahl (1985). He notes that in (23a) the determiner can have a reading where its restriction does not consist of the N', and where its matrix does not correspond to the VP: he says,

(23) a. Many SCANDINAVIANS have won the Nobel prize in literature

b. Most SCANDINAVIANS have won the Nobel prize in literature

can mean 'many that are winners of the NP in literature are Scandinavians.' He also observes that when we replace many with most, as in (23)b, such a 'switched' reading is not possible. 
(24) cannot be interpreted as 'most of those that have won the Nobel prize in literature are Scandinavians.'

Because Westerstahl assumes that the restriction necessarily corresponds to the $\mathrm{N}^{\prime}$ and the matrix to the VP, he concludes that many in (23a) is actually non-conservative:

(24) Conservativity: (Keenan and Stavi 1986) $[Q \mathrm{~A}] \mathrm{B}$ iff [Q A]B $\cap \mathrm{A}$

Given that conservativity is supposed to hold of all natural language determiners, the conclusion reached in Westerstahl is unexpected.

Going back to (23a) now, notice that the 'switched' reading actually only arises when Scandinavians is focused, as shown in (25)

Many SCANDINAVIANS have won the Nobel prize in literature

The switched reading is an instance of $f$-a quantification and (23a) has the LF in (26), where the focused predicate assumes the syntax of the main predicate and it becomes the matrix of the determiner:

(26) [E e: C (e) \& win the NP (e)] [many $x$ : things $(x, e) 0(x, e)] ~ E ~ e ':$ $R\left(e, e^{\prime}\right)$ \& SCANDINAVIANS $\left(x, e^{\prime}\right)$

Since $f$-a quantification only arises in weak NPs it is predicted that (23b), where the NP is necessarily strong, does not allow for a switched reading. Moreover, since restriction and matrix have been defined in purely syntactic rather than categorial terms, we maintain that many in (23a), and more generally the determiners of f-a NPs are conservative.

\section{Alternative semantics for $f-a$ readings?}

Clearly, the claim that in a $f-a$ readings the focused predicate serves as the syntactic main predicate of the sentence is strongly reminiscent of the traditional intuition that focus represents the main assertion of the sentence, cf. Prague school linguists, Löbner (1990).

This view of focus differs from that advocated in Rooth (1985). Under Rooth's conception, focus does not affect the predicational hierarchy of the sentence. Rather, it is viewed as creating a second, alternative semantic value, which, for simplicity's sake, I will refer to as the focus frame. The fact that focus can affect the truth conditions if embedded under certain operators is attributed not to focus as 
such, but to the meanings of these operators, which whose meaning is expressed in terms of focus and focus frame, cf. (6).

A question immediately arises as to whether this second view could not also account for $f$-a readings and why the one argued for here should be preferable? I think this view of focus could actually account for $\mathrm{f}$-a interpretations, for one could simply add weak determiners to the list of focus-sensitive operators. But if focus does not affect the syntax of the sentence, then it is not obvious how to maintain that determiners are conservative.

In an effort to secure the conservativity of determiners and at the same time keep an alternative view of focus, one could adopt a syntactically-minded version of Rooth's theory, eg. the view put forth in Partee (1991), where it is proposed that the focused predicate is mapped into the nuclear scope of a tripartite structure and the focus frame is mapped into the restrictive clause. If we extend this approach to also take into account cases of $f$-a quantification, we obtain the structure in (27) for the f-a reading of (7):

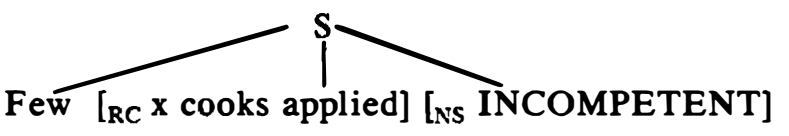

At first glance, (27) looks similar to the LF given earlier, essentially [E e: apply (e)] [few:x cook $(x, e)]$ INCOMPETENT $(x)$. There is an important difference, however. The tripartite structure cannot express that $f-a$ readings are licensed only inside the scope of the event operator. But failure to express this causes this view to derive a wrong logical form for (28) (Anna Szabolcsi (p.c.):

(28) Few INCOMPETENT cooks ever applied

(28) does not have a f-a reading for it cannot mean 'few cooks that ever applied were incompetent.' But the tripartite approach would predict that exactly this reading is available. This is shown in (29):

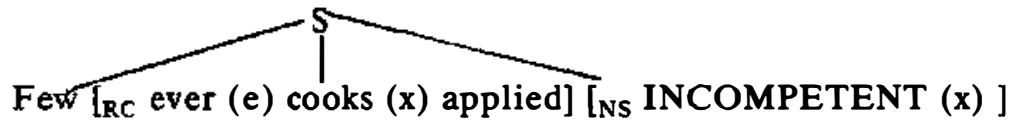

In (29) the negative polarity item ever associated to the event is licensed in the scope of $f e w$ and nothing barrs this structure. It remains unexplained why no $f$-a reading is available for (28).

Under the present analysis, on the other hand, f-a readings 
arise only inside the scope of the event operator. As a result, if we try to give the sentence a $\mathrm{f}$-a interpretation, the ever associated with event is not inside the scope of $f e w$ and fails to be licenced:

*Ever e: apply (e) [few x: cooks $(x, e)]$ INCOMPETENT (x)

In sum, it seems that the most straight-forward way to account for $\mathrm{f}-\mathrm{a}$ readings and to maintain that determiners are conservative is to take the view that focused predicates are syntactically the main predicates of their sentence and to NOT assume that quantified NPs are split apart into tripartite structures.

\section{The weak/strong contrast in terms of familiarity:}

The question that remains now concerns the weak-strong distinction. We have seen that because of the existence of $f-a$ readings it cannot be maintained that the strong/weak contrast coicides with whether determiners like $f e w$ and many are interpreted as symmetric or not. After all, when they introduce weak NPs with $f$-a readings, $f e w$ and many are proportional. As an alternative way of telling apart weak and from strong NPs, let us consider Enc's (1991) proposal that the relevant notion is partitivity.

Enc argues that strong NPs are 'familiar' in that they can be anaphorically related to an antecedent in the discourse, while weak NPs are 'novel'. Assuming that a definite description inside an NP is what allows for an anaphoric relation, she argues that strong but not weak NPs are (covert) partitives. (34) shows that familiarity is really the crucial divide between strong and the two types of weak NPs.

(34) The hotel fired all its employees. Few INCOMPETENT cooks reapplied.

To make sense out of the discourse one virtually has to relate the incompetent cooks talked about in the second sentence to the employees mentioned in the first, i.e. only a 'familiar' interpretation of the NP few INCOMPTENT cooks is sensible here. Notice now that the only reading of the NP we obtain in (34) is a strong one, meaning 'few of the incompetent cooks'. Both a reading of the NP where the determiner is symmetric and f-a reading ('few cooks that reapplied were incompetent') are clearly not available. This shows that familiarity succesfully tells apart strong NPs not only from 'regular' weak NPs, but also from f-a weak NPs. 
Let us then say that the determiners of strong NPs select a definite description as their complement, while the determiners introducing weak NPs do not. Formulating it in terms of the DPhypothesis, the determiners introducing strong NPs select for a DP in their complement, while the determiners introducing weak NPs select for an NP (cf. eg. Stowell 1989). If we now assume that the presence of a definite description creates a domain that is opaque to extraction, then we have an account of why strong NPs do not allow f-a readings: they do not allow for a focused predicate to be extracted. Notice that it is also exactly the group of strong NPs which does not allow for overt extraction and which exhibit the Specificity Condition (cf. Fiengo and Higginbotham 1980). We can then argue that the impossibilty of $f-a$ readings of strong NP is really the result of the Specificity Condition applying to extraction at LF.

\section{Conclusion:}

Looking at the effects of focus inside quantified NPs, we have seen that even with NPs introduced by few and many, the Definiteness Effect cannot be attributed to an ambiguity of the determiners, and is instead better accounted for in terms of (covert) partitivity. We have also seen arguments that show that all NPs, even weak ones, are quantificational in the traditional sense. The truthconditional effects of focus inside NPs have been accounted for by giving a concrete syntactic expression to the functionalist view that focus represents the main assertion of the sentence. A concrete proposal concerning another functionalist notion, namely that of topic, has also made it possible to explain scopal properties of subjects without attributing them to a lexical difference between SL and IL predicates.

\section{References}

Barwise, J. and R. Cooper (1981). 'Generalized Quantifiers and Natural Language.' Linguistics and Philoso phy 4, 159-219.

Beghelli, F. (1993) 'A Minimalists Approach to Quantifier Scope' NELS 23

Ben-Shalom, D. (1993) 'Object Wide Scope and Semantic Trees', paper presented at SALT 3.

Diesing, M. (1990) The Syntactic Roots of Semantic Partition. Ph.D. diss., U Mass, Amherst.

Dretske, F. (1972). 'Contrastive Statements' Philosophical Review, 
411-437.

Enc, M. (1991) 'The Semantics of Specificity' Linguistic Inquiry 22, $1-35$.

Fiengo, R. and J. Higginbotham (1980). 'Opacity and NP' Linguistic Analysis 7, 397-421.

Gueron, J. 'On the Syntax and Semantics of PP Extraposition'Linguistic Inquiry 11, 637-677.

Higginbotham, J. (1987) 'Indefiniteness and Predication' in Reuland E. and A.ter Meulen The Representation of (In)definiteness MIT Press, Cambridge, Mass.

Keenan, E. and Stavi (1986). 'A Semantic Characterization of Natural Language Determiners' Linguistics and Philosophy 9, 253-326.

Kratzer, A. (1989) 'Stage and Individual Level Predicates' Papers on Quantification, $\mathrm{U}$ Mass, Amherst.

Krifka, M. (1992) 'A Framework for Focus-Sensitive Quantification', in Proceedings from SALT 2, Ohio State University Working Papers in Linguistics 40.

Löbner, S. (1990) Wahr neben Falsch Max Niemeyer, Tübingen.

May, R. (1985) Logical Form: Its Structure and Derivation. MIT Press Cambridge, Mass.

Milsark, G. (1977) 'Towards an Explanation of Certain Peculiarities of the Existential Construction in English' Linguistic Analysis 3, 1-29.

Moltmann, F. (1990) 'Scrambling in German and the Definiteness / Specificity Effect' ms. MIT.

Partee, B. (1988) 'Many Quantifiers' In Proceedings of ESCOL

Partee, B. (1991) 'Topic, Focus and Quantification', in Proceedings from $S A L T 1$ Cornell Working Papers in Linguistics 10.

Raposo, E. and J. Uriagereka (1990) 'Long-distance Case Assignment' Linguistic Inquiry 21

Rooth, M. (1985) Association with Focus. Ph.D. diss., U Mass, Amherst.

Schein, B. (1992) Plurals and Events. ms. USC.

Stowell, T. (1989). 'Subjects, Specifiers, and X-Bar Theory' in Baltin, M. and A. Kroch eds. Alternative Conceptions of Phrase Structure Univ. of Chicago Press, Chicago, IL.

Westerstahl, D. (1985). 'Logical Constants in Quantifier Languages' Linguistics and Philosophy 8, 387-413.

Dept. of Linguistics

Univ. of Southern California

Los Angeles, CA 90089-1693

herburge@scf.usc.edu 